\title{
The Use of High-Power Electromagnetic Pulses on Bacteria and Viruses
}

\author{
Academician Yu. V. Gulyaev ${ }^{a, *}$, I. V. Taranov ${ }^{a, * *}$, \\ and Corresponding Member of the RAS V. A. Cherepenin ${ }^{a, * * *}$
}

Received May 12, 2020revised May 12, 2020; accepted May 18, 2020

\begin{abstract}
The hypothesis about the possibility of using strong electromagnetic pulses for effective action on bacteria and viruses is discussed. A simple coronavirus model that allows us to obtain in a quasi-static approximation estimates of the electric field strength is analyzed.
\end{abstract}

Keywords: electromagnetic pulse, effects on viruses, pondermotive force

DOI: $10.1134 / \mathrm{S} 1028335820070034$

The problem of the effect of electromagnetic waves on biological media or objects has a relatively long history. In relation to medical aspects, various diagnostic methods, physiotherapy, and determination of permissible radiation doses were studied when working with radiating devices used for communication, radar, or other applications. In recent years, work has appeared on the effects of microwaves on bacteria and viruses. Thus, in [1], a mechanism of resonant action on viruses based on the transformation of electromagnetic waves into acoustic ones is proposed. This communication is about nonthermal effects on biological objects of powerful electromagnetic pulses, the characteristic frequencies of which lie in the microwave range and the duration of which is in the nanosecond range. Such pulses were used to extract precious metals from refractory ores [2], to open vesicles in solving the problem of targeted drug delivery $[3,4]$, and to determine effects on the cell membrane [5], as well as in some other applications. The physical idea of these studies, in contrast to their implementation, is quite simple.

When exposed to a pulse with high field strength $\mathbf{E}$, charges arise on the medium with electrophysical inhomogeneities at the interfaces, which provoke various, rather complex physical phenomena, such as micro-breakdowns, mechanical deformation, and cracking under the action of pondermotor forces and

\footnotetext{
${ }^{a}$ Kotelnikov Institute of Radioengineering and Electronics, Russian Academy of Sciences, Moscow, Russia

*e-mail:gulyaev@cplire.ru

**e-mail:ivt@cplire.ru

***e-mail: cherep@cplire.ru
}

other processes leading to modification of the medium. In particular, when exposed to biological objects with sizes of $0.1-1 \mu \mathrm{m}$, which is characteristic of viruses and bacteria, their destruction is possible. It should be noted that the effectiveness of the effect depends on the shape of the bio-object; in particular, the COVID-19 virus has characteristic nano-protrusions [6-9], which facilitate penetration into the body cell. As is known, the field strength near micro- or nano-points increases, which should lead to damage at a lower value of the external field strength.

Here are some estimates for a fairly simple model that demonstrates a pondermotor effect. As a model of the spiny process containing receptor-binding proteins and forming the "solar corona" of the virus, we choose a dielectric ellipsoid. Consider the problem of the occurrence of torque $N$ pondermotor forces applied to a spherically asymmetric dielectric object with a dielectric constant $\varepsilon_{c}$ surrounded by a dielectric medium with a dielectric constant $\varepsilon_{m}$ when exposed to an external electric field $\mathbf{E}_{0}$. The spherically asymmetric (elongated) dielectric body considered can simulate the behavior of the spikelike element of the "crown" of the virus, due to external electrical exposure. Air can be considered as the environment (in this case $\varepsilon_{m}=1$ ) or another weakly conducting medium. For simplicity, we assume that the shape of the spineshaped process of the virus has the shape of an elongated ellipsoid with the main semi-axes $a>b \geq c$ (Fig. 1).

In this case, the torque moment $N$ applied to the elongated ellipsoidal body tending (in the case $a>b \geq c$ ) to turn the main axis in the direction of the electric field $\mathbf{E}_{0}$ : 


$$
N=\frac{V E_{0}^{2}}{8 \pi} \frac{\left(\varepsilon_{c}-\varepsilon_{m}\right)^{2}(1-3 n(a, b, c))}{\left(\varepsilon_{m}+\varepsilon_{c}+\left(\varepsilon_{c}-\varepsilon_{m}\right) n(a, b, c)\right)\left(\varepsilon_{m}+\left(\varepsilon_{c}-\varepsilon_{m}\right) n(a, b, c)\right)} \sin 2 \vartheta,
$$

where $\vartheta$ is the angle between $\mathbf{E}_{0}$ and the largest semiaxis of the ellipsoid, $V$ is the ellipsoid volume, and $n(a$, $b, c)$ is the depolarization coefficient

$$
n(a, b, c)=\frac{3 V}{8 \pi} \int_{0}^{\infty} \frac{d \xi}{\left(\xi+a^{2}\right) \sqrt{\left(\xi+a^{2}\right)\left(\xi+b^{2}\right)\left(\xi+c^{2}\right)}},
$$

which in the case of an ellipsoid of revolution $(a>b=c)$ can be represented in elementary functions:

$$
n=\frac{1-e^{2}}{e^{2}}\left(\frac{1}{2 e} \ln \frac{1+e}{1-e}-1\right),
$$

where $e=\sqrt{1-\frac{b^{2}}{a^{2}}}$ is the eccentricity of the ellipsoid of revolution ( $n=\frac{1}{3}$ for a sphere and $n \ll 1$ for a very elongated body $b \ll a$ ).

In the case when the magnitude of the torque of forces (1) is comparable with the energy of chemical bonds $\epsilon_{0}\left(\epsilon_{0} \approx 1 \mathrm{eV}\right)$

$$
N^{c r}=\in_{0},
$$

we can expect a destructive effect of the rotating elliptical object on the connection with the nearest structural elements of the virus. Condition (3) determines the critical value of the electric field strength

$$
E_{0}^{(c r)}=2\left[\frac{2 \pi \epsilon_{0}}{V} \frac{\left(\varepsilon_{m}+\varepsilon_{c}+\left(\varepsilon_{c}-\varepsilon_{m}\right) n(a, b, c)\right)\left(\varepsilon_{m}+\left(\varepsilon_{c}-\varepsilon_{m}\right) n(a, b, c)\right)}{\left(\varepsilon_{c}-\varepsilon_{m}\right)^{2}(1-3 n(a, b, c))}\right]^{\frac{1}{2}},
$$

at which the magnitude of the torque becomes sufficient for the destructive action of the spikelike processes. For the characteristic values of the parameters of the problem under consideration $\varepsilon_{m}=1$ (air), $\varepsilon_{c}=3$, $\epsilon_{0}=1 \mathrm{eV}$, the largest ellipsoid size is $40 \mathrm{~nm}, \frac{a}{b}=0.5$, and the critical field value takes on the value

$$
E_{0}^{(c r)}=450 \mathrm{kV} / \mathrm{cm} \text {. }
$$

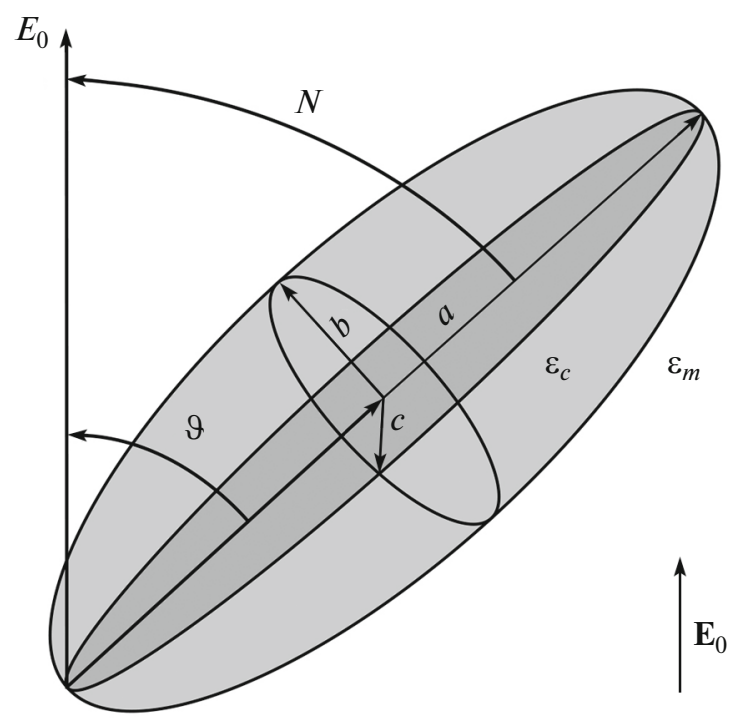

Fig. 1. Torque applied to an ellipsoidal body.
It is clear from the above estimate that a similar, at first glance, fairly high value of the electric field for biological objects is, rather, the upper estimate due to the assumption that the object is smooth. However, even when implemented, it will not have a noticeable effect on more homogeneous cellular structures of biological objects in the case of ultrashort exposure with a pulse duration on the order of $10^{-9} \mathrm{~s}$, since the electric strength of the media increases significantly with shortening the pulse duration [10].

The electrophysical properties of biological systems, especially viruses, are generally unknown. The criteria for the optimal intensity of the electric component of the field, the duration of the pulses, and their repetition rate must be selected experimentally.

The example of the current COVID-19 pandemic shows the need for not only chemical, but also physical sterilization methods. The proposed electromagnetic method compares favorably with other methods in its simplicity and the absence of long-term side effects.

\section{FUNDING}

This work was performed as part of a state assignment, project no. AAAA-A19-119041590070-1, and supported by the Russian Foundation for Basic Research, project no. 1829-02094.

\section{REFERENCES}

1. Szu-Chi Yang, Huan-Chun Lin, Tzu-Ming Liu, JenTang Lu, Wan-Ting Hung, Yu-Ru Huang, Yi-Chun Tsai, Chuan-Liang Kao, Shih-Yuan Chen, and Chi- 
Kuang Sun, Sci. Rep. 5, 18030 (2016). https://doi.org/10.1038/srep18030

2. V. A. Chanturiya, Yu. V. Gulyaev, V. A. Cherepenin, V. D. Lunin, I. Zh. Bunin, V. A. Vdovin, A. V. Korzhenevskii, Dokl. Akad. Nauk 366 (5), 680-683 (1999).

3. Yu. V. Gulyaev, V. A. Cherepenin, I. V. Taranov, V. A. Vdovin, A. A. Yaroslavov, V. P. Kim, and G. B. Khomutov, J. Commun. Technol. Electron. 61 (1), 56-60 (2016).

4. Yu. V. Gulyaev, V. A. Cherepenin, I. V. Taranov, V. A. Vdovin, A. A. Yaroslavov, V. P. Kim, and G. B. Khomutov, J. Commun. Technol. Electron. 63 (2), 158-162 (2018).

5. N. D. Devyatkov, Z. S. Chernov, O. V. Betskii, T. A. Noskova, and A. V. Putvinskii, Biofizika 28 (3), 558-560 (1982).
6. R. H. Yan, Y. Y. Zhang, Y. N. Li, L. Xia, Y. Y. Guo, and Q. Zhou, Science 367 (6485), 1444-1448 (2020). https://doi.org/10.1126/science.abb2762

7. L. Zhang, D. Lin, X. Sun, U. Curth, Ch. Drosten, L. Sauerhering, S. Becker, and K. Rox, Science 368 (6489), 409-412 (2020). https://doi.org/10.1126/science.abb3405

8. R. N. Kirchdoerfer, Ch. A. Cottrell, N. Wang, J. Pallesen, H. M. Yassine, H. L. Turner, K. S. Corbett, B. S. Graham, and S. Jason, Nature 531 7592), 118$121(2016)$. https://doi.org/10.1038/nature 17200

9. M. Gui, W. Song, H. Zhou, J. Xu, S. Chen, Y. Xiang, and X. Wang, Cell Res. 27, 119-129 (2017). https://doi.org/10.1038/cr.2016.152

10. G. A. Mesyats and M. I. Yalandin, Usp. Fiz. Nauk 175, 225-246 (2005). https://ufn.ru/ru/articles/2005/a. 